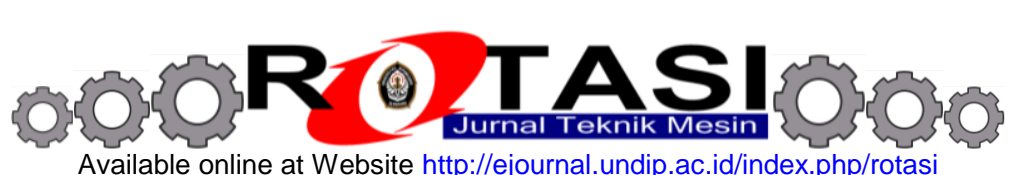

Available online at Website http://ejournal.undip.ac.id/index.php/rotasi

\title{
SUMMARY OF THE RECENT DEVELOPED TECHNIQUES FOR MACHINE HEALTH PROGNOSTICS
}

\author{
*Achmad Widodo, Wahyu Caesarendra \\ Department of Mechanical Engineering, Diponegoro University \\ Jl. Prof. Sudharto,Tembalang, Semarang 50275, Indonesia \\ *Email: awid@undip.ac.id
}

\begin{abstract}
This paper reviews relatively new developed techniques for machine health prognostics system. The prognostics assessment of machines is an important consideration for determining the remaining useful life (RUL) of machine components and prediction of future state of machines. The developed system has employed several approaches of machine health prognostics strategy such as data-driven, physical-based, and probability-based methods. The method of solution implemented artificial intelligence techniques including support vector machine (SVM), relevance vector machine (RVM), Dempster-Shafer theory, decision tree, particle filter, and autoregressive moving average/ generalized autoregressive conditional heteroscedasticity (ARMA/GARCH). Case studies of machine health prognostics are also presented to show the plausibility of the developed systems. Finally, this paper summarizes the research finding and directions of machine health prognostics system.
\end{abstract}

Keywords: artificial intelligence, machine prognostics, remaining useful life

\section{INTRODUCTION}

In today's highly competitive marketplace, industries strive to minimize their capital and operational costs by trying to utilize the whole life cycle of their machinery without sacrificing human, production, or environmental safety. Condition-based maintenance (predictive maintenance) is most useful in predicting equipment failure and avoiding unnecessary maintenance activities. Prognostics (also called prognosis) is an inherent component of condition-based maintenance. Prognosis is the ability to assess the current health of a part and predict into the future the health of a part for a fixed time horizon or predict the time to failure. Being able to perform reliable prognostics is the key to conditionbased maintenance. Prognosis is critical to the machine for improving safety, planning missions, scheduling maintenance costs and downtime.

Prediction of RUL of machine and machine degradation are objectives of prognosis assessment. The efforts to accurately estimate such objectives have been studied for recent decade. However, this topic is still interesting and relevance to be studied for contributing engineering asset management task. A number of techniques and methods have been extensively studied in literature related to the way to assess the degradation parameter or deviation parameter. The published papers which report the review of machine prognosis are presented in Refs. [1-3]. Jardine et al. [2] explained, the approaches to prognosis fall into three main categories: statistical approaches, artificial intelligent approaches and model-based approaches. Among all, artificial intelligent approaches are more popular. Artificial intelligent techniques also known data-driven prognosis is a method to calculate the model based on training process of prior data, and then predict the future state using one-step or multi-step ahead prediction. Physic-based also known model-based prognosis is a method to predict the future condition by modeled the failure model or damage model e.g. crack model.

This paper presents research output of developed prognosis techniques based on data-driven, model-based and probability-based techniques. The techniques presented in this paper are relatively new contribution to the machine health prognosis that utilizes intelligent tools such as support vector machine (SVM), relevance vector machine (RVM), decision tree, neuro-fuzzy system, Dempster-Shafer, autoregressive moving average (ARMA), and generalized autoregressive conditional hetero-scedasticity $(\mathrm{GARCH})$. For the probability-based, the SVM and RVM are combined by survival analysis, Cox proportional hazard model and logistic regression in order to assess the failure degradation and prediction from incipient failure until final failure occurred. The basic theory of intelligent tools will be firstly reviewed then followed by additional theory for probability-based. Finally, several case studies will be presented to show the plausibility of proposed intelligent machine health prognosis.

\section{THE DEVELOPED TECHNIQUES}

We have developed various prognosis methods based on data-driven, model-based and probability-based. The following will review the prognosis method solutions and their implementations.

\subsection{Data-driven method}

Data-driven prognosis method attempts to derive the models directly from the data collected routinely from condition monitoring devices. They are built based on historical record and produce prediction outputs directly in terms 
of condition monitoring data. The developed methods of prognosis in our laboratory employed several artificial intelligent techniques are presented as follows:

2.1.1. Support vector machine (SVM)

The theoretical foundation of SVM was developed by Vapnik [4]. Our first study of prognosis was application of SVM for predicting future state condition of machine reported in Ref. [5]. The method of implementation is described in Figure 1 as follows

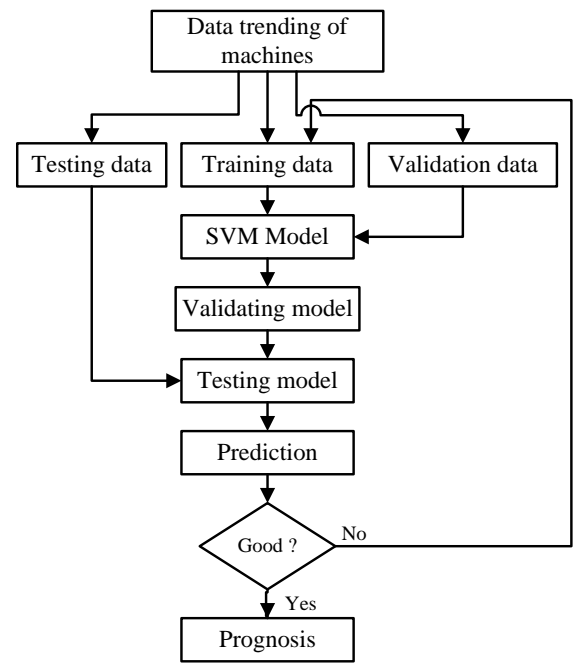

Figure 1. SVM for prognostics implementation.

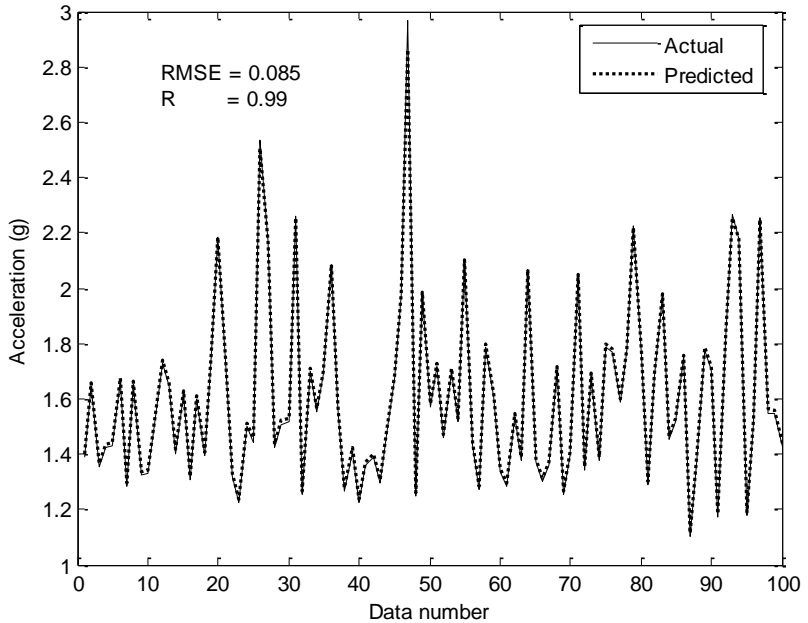

Figure 2. Prediction of envelope acceleration data.

The data used in this experiment is data trending of machine based on vibration signal which contains data histories of machine until faults occurred. Then, the data trending is divided into three parts: training data, validation data and testing data. The training and validation data are used to build the model for machine fault prognosis system, while the testing data is used to test the validated model. After model validation, the tested model will be obtained. The tested model is used to predict the future data that is never used for training and validation. The quality of prediction result is measured by performance measures e.g., root-means square error $R M S E$ and correlation coefficient $R$. Prognosis system is obtained if the prediction is successful and passed the user defined criterion of performance measures. This method was successfully implemented in prediction the future state of low methane compressor based on acceleration data (Figure 2).

\subsubsection{Decision tree (DT)}

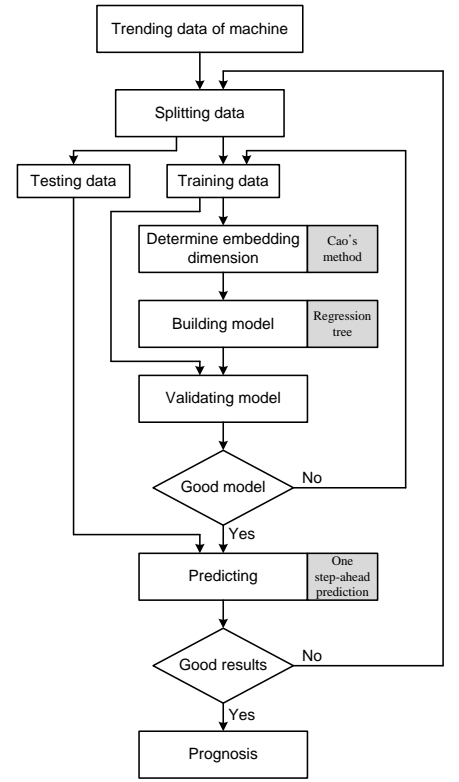

Figure 3. The prognostics method by DT.

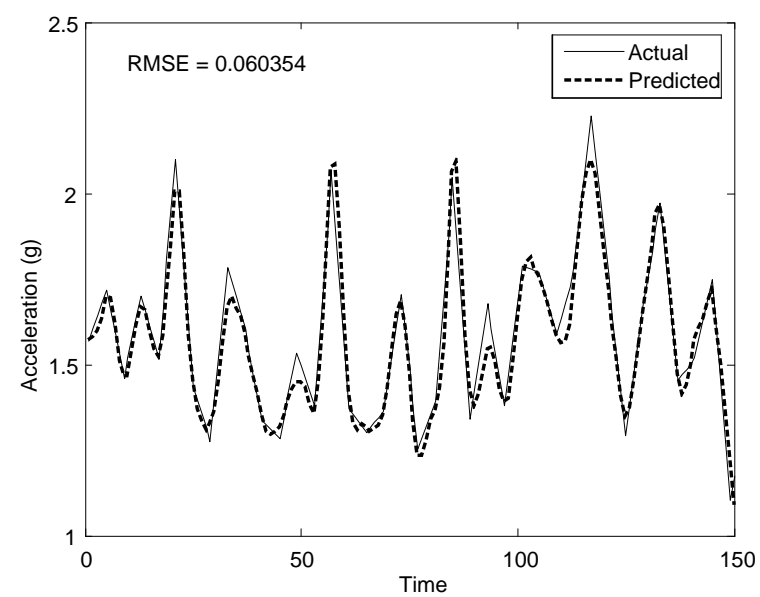

Figure 4. Prediction of envelope acceleration data. 
Our success of DT implementation for machine prognostics has been reported in [6]. Such paper proposes a method to predict the future conditions of machines based on one-step-ahead prediction of time-series forecasting techniques and regression trees. In that study, the embedding dimension is firstly estimated in order to determine the necessarily available observations for predicting the next value in the future. This value is subsequently utilized for the predictor which is generated by using regression tree technique. The proposed method is mainly similar with previous technique that consists of four steps: data acquisition, data splitting, training-validating and predicting. The flowchart of method is depicted in Figure 3. The proposed method was successfully applied to predict similar machine as previous example by SVM with different number of training and testing data. The result prediction is presented in Figure 4.

\subsubsection{Dempster-Shafer regression (DSR)}

The DSR technique was adopted as a new time-series prediction model for machine prognostics purpose. DSR or evidence regression (EVREG) was introduced by Renaud and Denœux [7] by adopting the subjectivist, nonprobabilistic view of 'Smets' transferable belief model (TBM) [8]. Basically, the method considers each training sample from the neighborhood of the input vector as a piece of evidence regarding the value of the output. The pieces of evidence are discounted as a function of their distance to the input vector, and pooled using Dempster's rule of combination. This technique was successfully applied to estimate future state condition of low-methane compressor using multi-step ahead prediction. The result is presented in Figure 5.

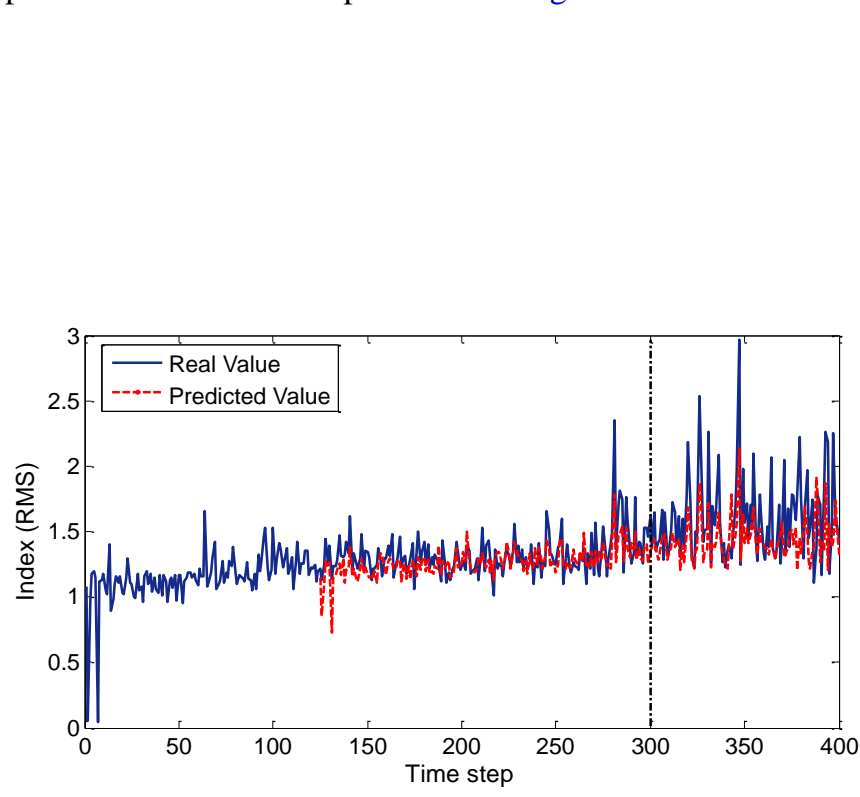

Figure 5. Prediction of acceleration trend by DSR.

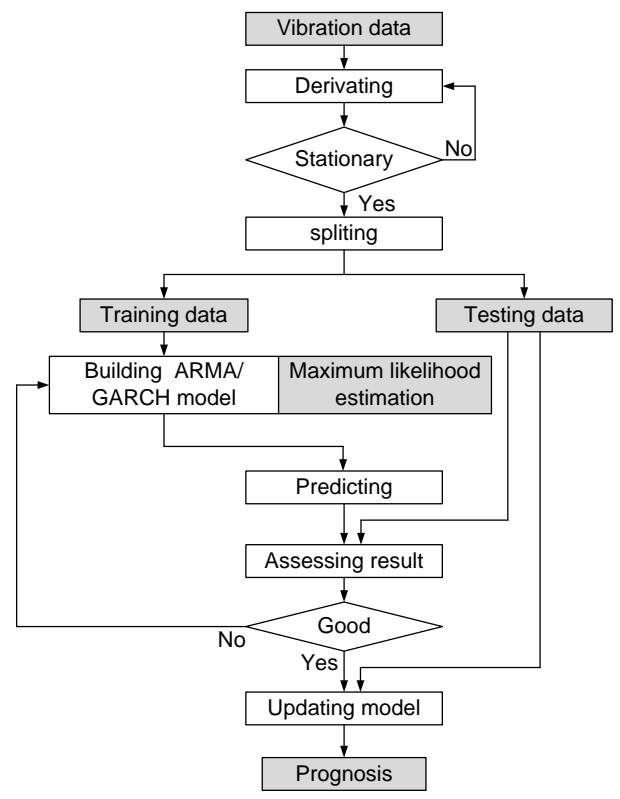

Figure 6. The ARMA/GARCH prediction model.

\subsubsection{ARMA/GARCH}
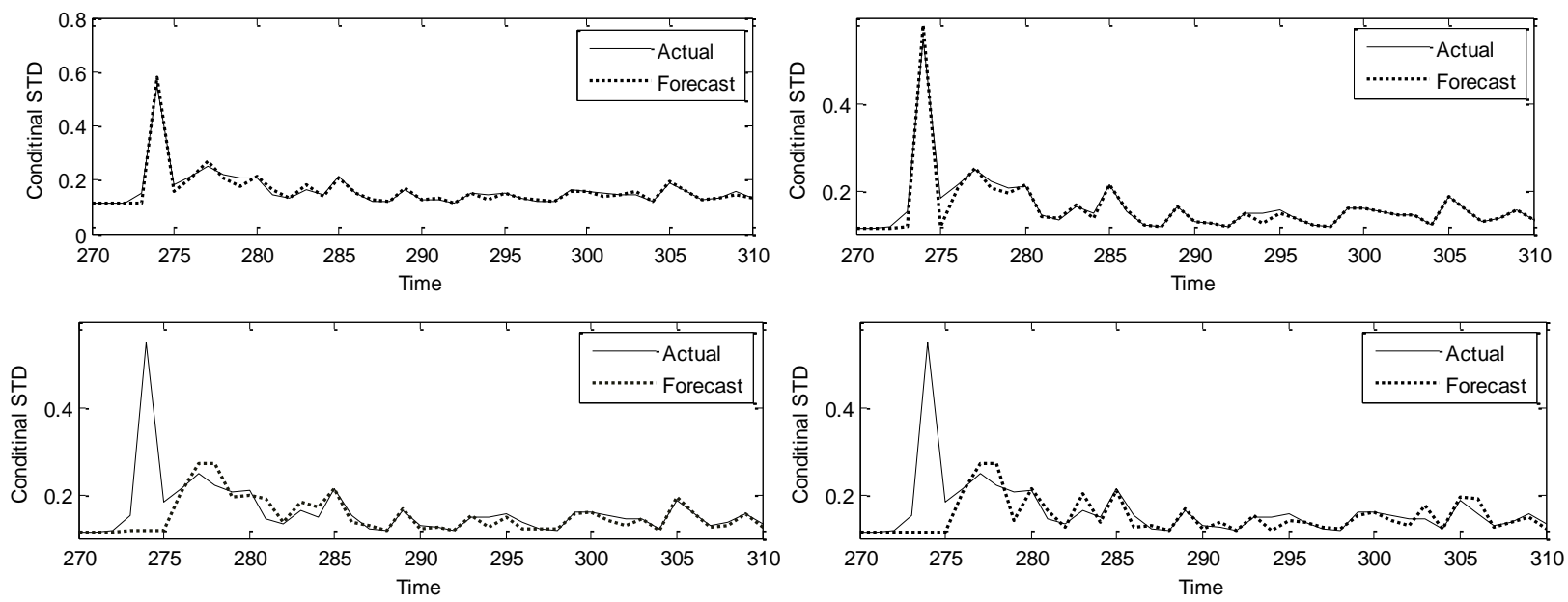

Figure 7. The conditional standard deviation forecasts of innovations using $1 \sim 4$ step-ahead. 
We have also developed ARMA/GARCH for prognostics health condition of machine [9]. The main idea of implementation is to employ the linear ARMA model and nonlinear GARCH model to explain the wear and fault condition of machine, respectively. The successful outcomes of the ARMA/GARCH prediction model can give obvious explanation for future states of machine which enhance the worth of machine condition monitoring as well as conditionbased maintenance in practical applications. The proposed method is summarized in the flowchart as depicted in Figure 6.

First, the acquired time-series data e.g., vibration data needs to be made up as stationary data before inputting into ARMA/GARCH model. The stationer data is then divided into two parts: training data and testing data. Training data is used to build forecasting model and to determine the parameters for the ARMA/GARCH model by using the maximum likelihood estimation. Testing data is used to assess the performance capability of the model. Training data is used. After the adequate model is estimated, forecasting is performed to predict the future condition of machine health. The final step is updating the model after one time step prediction to achieve more accurate results and capable to multistep ahead prediction. The ARMA/GARCH based prognosis system has been successfully applied to predict health condition of low-methane compressor similar with previous example presented in Figure 7.

\subsection{Model-based method}

Model-based or known as physic-based model typically involve building technically comprehensive mathematical models to describe the physic of the system and failure modes, such as crack propagation and spall growth modelled by Paris law [10]. These models attempt to combine system specific knowledge, defect growth formula and condition monitoring data to provide 'knowledge rich' prognosis output.

\subsubsection{Particle filter (PF)}

$\mathrm{PF}$ is a technique for simulating samples and then approximates the target distribution by weighting these samples using appropriately importance weight [11]. PF essentially consists of three computational steps. Firstly is sampling that presents a set of random samples (particles) of unknown state are generated based on the given sampling function. Secondly is weight calculation based on the observation, and then the importance weights are estimated from those particles. Thirdly is re-sampling that involves redrawing particles from the same probability density based on some function of the particle weights.

In our developed system, PF has been applied to crack growth rate prediction and estimation of the residual life in cracked specimens of the pressure vessel steel (SPV50) based on random crack propagation data. In our experiment, ten crack propagation data have been acquired by fatigue crack growth testing under constant amplitude loading. To reduce the randomness phenomena, three probability density functions are utilized. Crack propagation parameter is calculated using empirical Paris law equation. A numerical approach by FEM in Figure 8 is used to determine the stress intensity factor (SIF). Furthermore, PF is employed as prediction method. Results from the analysis and tests agreed well and can be used for residual life prediction of final failure (Figure 9).

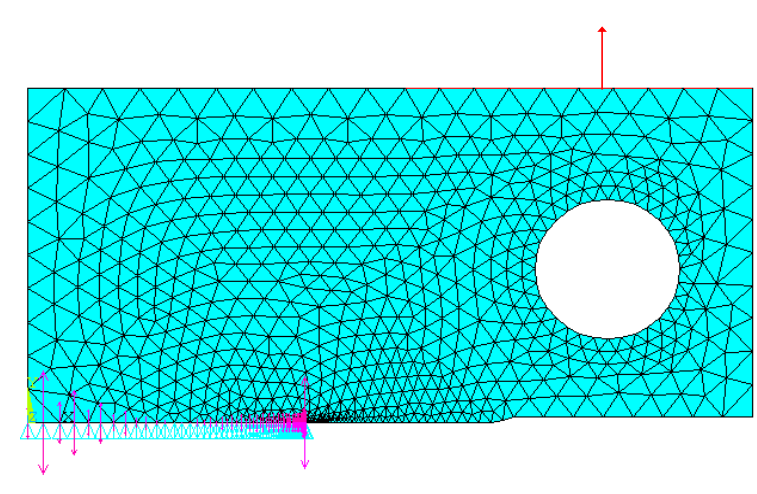

Figure 8. FEM model for SIF determination.

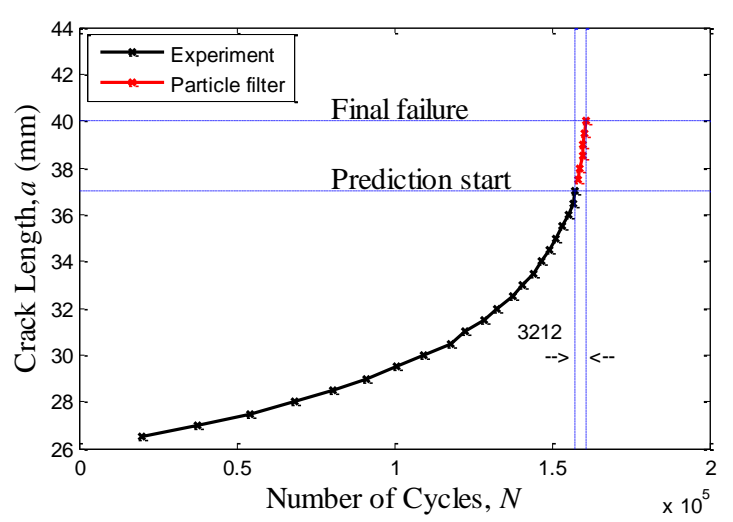

Figure 9. The result of crack prediction.

\subsection{Probability-based method}

This method concerns with fitting the probability failure distribution to historical data. The logic extension to this method is the correlation of failure event history with more specific health condition data. It estimates the life of an average component under average usage condition.

\subsubsection{Survival analysis and relevance vector machine (RVM)}

In our development, probability-based method is presented using survival probability estimation of machine element under operation e.g., rolling element bearing by Kaplan-Meier (KM) method [12] and conditional probability density function (PDF). This method was combined by historical condition monitoring data e.g., vibration data, and 
integrated by intelligent system through RVM regression for failure prediction [13]. In our proposed system, condition monitoring data is considered to be integrated with reliability analysis to enable prognostic system that is longer-range system. The censored data of historical units usually rare to be considered as prognostic input data and it has also not been fully utilized. Whereas this phenomenon is very common in the practice that the system does not contain of only single unit but a population of units. The training inputs for RVM are generated from experimental bearing defect degradation data that involves censored data [14]. Target vectors are survival probability obtained from survival analysis by using KM and conditional PDF estimators. The proposed method and the result are depicted in Figure 10 and Figure 11, respectively.

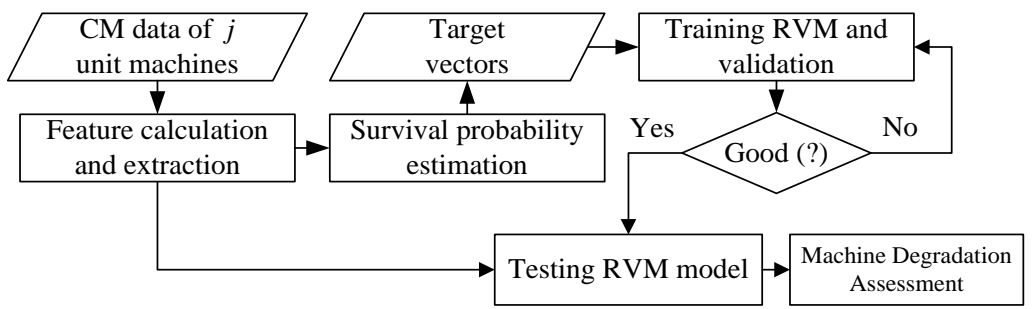

Figure 10. Machine degradation assessment method by RVM.

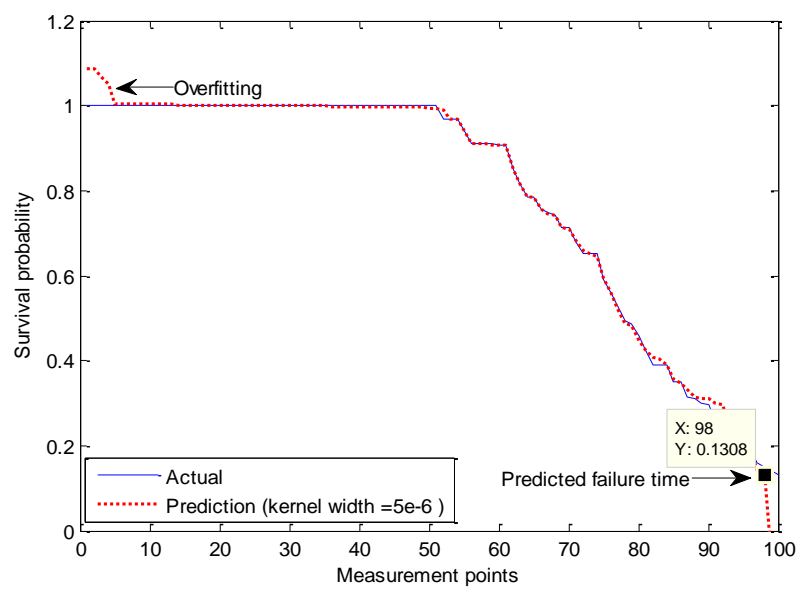

Figure 11. RVM prediction of bearing defect degradation.

\subsubsection{Cox-proportional hazard (CPH) and SVM}

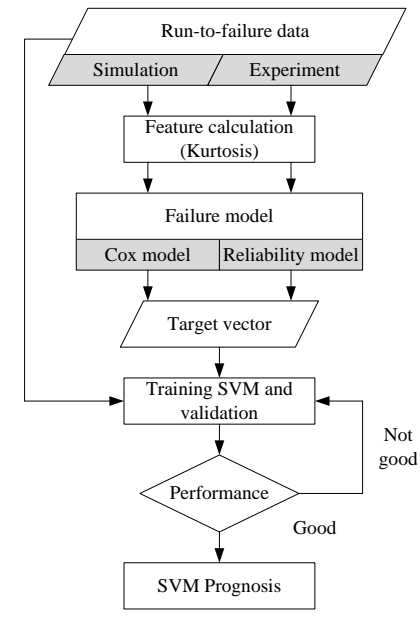

Figure 12. $\mathrm{CPH}$ and SVM prognostics method

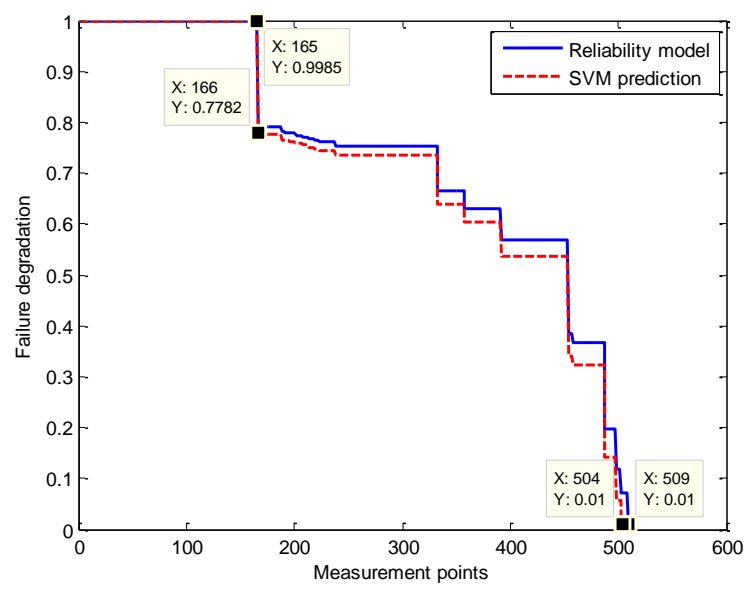

Figure 13. Failure degradation prediction by CPH-SVM

In this case, we developed prognosis system based on calculation of failure rate as the degradation parameter using CPH model and reliability theory of condition monitoring data. First, the degradation parameter is obtained by $\mathrm{CPH}$ model where baseline hazard $h_{0}(t)$ is assumed having Weibull probability. Weibull parameters are then determined by two parameters Weibull probability plot. In our study, we used rolling element baring vibration feature namely 
kurtosis as an incorporating explanatory variable or covariate. SVM is selected for intelligent prognostics tool where its training input and target are kurtosis of vibration signal and failure degradation, respectively. This technique has been employed to predict failure degradation of rolling element bearing using the data as presented in previous example. The method of prognosis degradation and the result are presented in Figure 12 and Figure 13, respectively.

\subsubsection{Logistic regression (LR) and relevance vector machine (RVM)}

In our development, we proposed prognosis technique based on LR in order to assess the failure degradation and prediction from incipient failure until final failure occurs [15]. LR is a variation of ordinary regression method which is used when the dependent variable is a dichotomous variable (which is usually represented the occurrence or nonoccurrence of some output event, usually coded as 0 and 1).

The goal of LR is to find the best fitting model to describe the relationship between the dichotomous characteristic of the dependent variable and a set of independent variables [16]. The LR was combined by RVM to perform intelligent prognosis system. This method has been applied to predict failure degradation of rolling element bearing as presented in the previous example. The LR calculation and result of prediction are presented in Figure 14 and Figure 15, respectively.

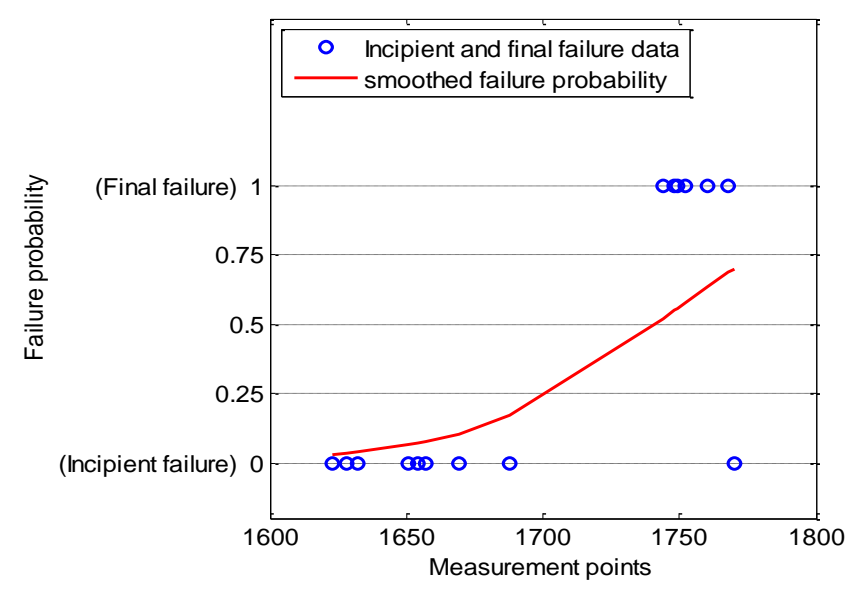

Figure 14. Logistic regression of data.

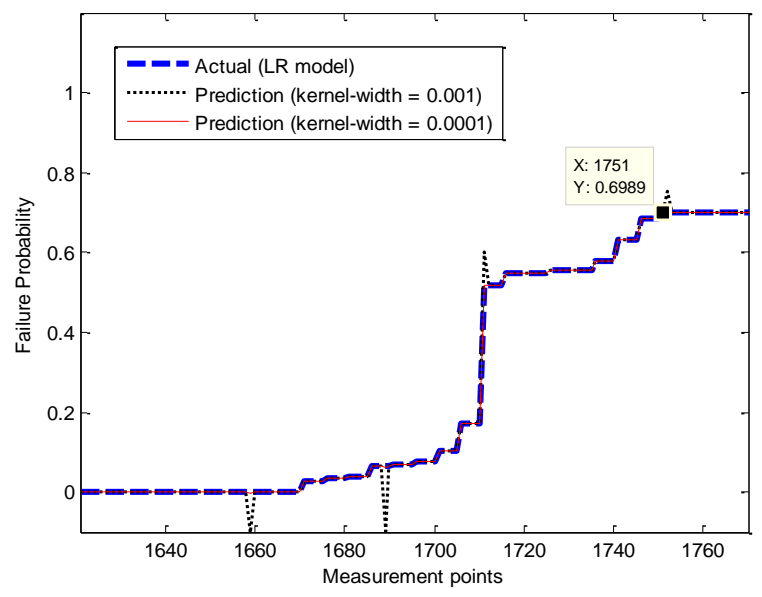

Figure 15. The result of degradation prediction.

\section{CONCLUSION}

This paper summarizes and reviews the research outcomes of machine prognosis study which had been conducted by authors and their team in IML-PKNU, Korea. The developed methods have employed various techniques for estimating failure degradation of machines being studied. Moreover, our developed systems concern with implementation of intelligent systems to obtain high accuracy in predicting health condition of machines. Even though many researchers have been conducted similar research of prognosis, but this area is still open and interesting to be studied. The effort to find research finding of machine prognosis that are effective and reliable in application should be emphasized.

\section{ACKNOWLEDGMENT}

This paper is a tribute to the late of Prof. Bo-Suk Yang from Pukyong National University, South Korea who taught the authors methods of machine fault diagnostics and prognostics. Authors also would like to thank to Dr. Niu Gang, Dr. Tran Van Tung, Mr. Hong Thom Pham for their contribution and cooperation.

\section{REFERENCES}

[1] Khotamasu R, Huang SH, and Ver Dui WH. System health monitoring and prognostics - A review of current paradigms and practices. International Journal of Advanced Manufacturing Technology 1996;28:1012-1024.

[2] Jardine AKS, Lin D, and Banjevic D. A review on machinery diagnostics and prognostics implementing conditionbased maintenance. Mechanical Systems and Signal Processing 2006;20:1483-1510.

[3] Heng A, Zhang S, Tan ACC, Mathew J. Rotating machinery prognostics: State of the art, challenges and opportunities. Mechanical System and Signal Processing 2009;23(3):724-739.

[4] Vapnik V. The nature of statistical learning theory. New York: Springer-Verlag;1995.

[5] Yang BS, Widodo A. Support vector machine for machine fault diagnosis and prognosis. Journal of System Design and Dynamics 2008;2(1):12-23. 
[6] Tran VT, Yang BS, Oh MS, Tan ACC. Machine condition prognosis based on decision trees and one-step-ahead prediction. Mechanical System and Signal Processing 2008;22:1179-1193.

[7] Petit-Renaud S, Denœux T. Nonparametric regression analysis of uncertain and imprecise data using belief functions. International Journal of Approximate Reasoning 2004;35(1):1-28.

[8] Smets P, Kennes R. The transferable belief model. Artificial Intelligence 1994;66:191-243.

[9] Pham HT, Yang BS. Estimation and forecasting of machine health condition using ARMA/GARCH model. Mechanical Systems and Signal Processing 2010;24(2):546-558.

[10] Paris PC, Erdogan F. A critical analysis of crack propagation laws. ASME Journal of Basic Engineering 1963;85:528-534.

[11] Doucet A, DeFreitas N, Gordon N. Sequential Monte Carlo in practice. New York: Springer-Verlag;2000.

[12] Kaplan EL, Meier P. Nonparametric estimation from incomplete observations. Journal of the American Statistical Association 1958;53:457-481

[13] Tipping ME. Sparse Bayesian learning and the relevance vector machine. Journal of Machine Learning Research 2001;1:211-244.

[14] Lee J, Qiu H, Yu G, Lin J, Rexnord Technical Services. 'Bearing data set', IMS, University of Cincinnati. NASA Ames Prognostics Data Repository, NASA Ames, Moffett Field, CA 2007. (see also: http://ti.arc.nasa.gov/project/prognostic-data-repository, accessed: December 11, 2009).

[15] Caesarendra W, Widodo A, Yang BS. Application of relevance vector machine and logistic regression for machine degradation assessment. Mechanical Systems and Signal Processing 2009; in press, doi:10.1016/j.ymssp.2009.10.011

[16] Yan J, Koc M, Lee J. A prognostic algorithm for machine performance assessment and its application. Production Planning and Control 2004;15(8):796-801. 